\title{
Resonant diffraction gratings with polarization-dependent efficiencies
}

\author{
Julian Wüster ${ }^{1, *}$, Patrick Feßer ${ }^{1}$, Arne Behrens ${ }^{1}$, and Stefan Sinzinger ${ }^{1}$ \\ ${ }^{1}$ Technische Universität Ilmenau, Center for Micro- and Nanotechnologies, FG Technische Optik, Am Helmholtzring 1, 98693 Ilmenau
}

\begin{abstract}
Subwavelength-structures with different fill factors in the lateral dimensions result in unique phase shifts for the different polarization states of transmitted light.

By using this additional degree of freedom for diffractive optical elements, we yield additional functionalities for compact optical systems with DOEs.

As a fully operable example we present a binary subwavelength-grating which acts as a polarizing beamsplitter for TE- and TM-polarization over a wide range of incidence angles. We show our design approach, the manufacturing process with Soft-UV-Nanoimprint-Technology, as well as experimental results. We will also lay out possibilities for the design and application of multilevel polarization-functionalized gratings.
\end{abstract}

\section{Introduction}

Polarizing beam splitters are classically realized with dielectric coatings or birefringent materials. Alternatively, beam splitters can also consist of high-frequency gratings (single-order gratings) under Bragg (or Littrow) incidence [1] or subwavelength-structures, e.g. under an incidence of $45^{\circ}$ [2]. For a compact in-line measurement system it is necessary to achieve a beamsplitting functionality while maintaining one optical axis over the whole system. This is possible in systems using a diffraction grating as shown in [3] and [4] which uses the $O^{\text {th }}$ order for illumination and as the light reflected from the surface-to-be-measured passes the grating again, the higher orders are used for the detection. For this it is advantageous to design a grating, which has low diffraction efficency in the direction of illumination and high efficiency for the reflected light. The distinction is possible with the help of a quarterwaveplate switching between TE- or TM-polarization, respectively.

\section{Design approach}

The basic idea is to combine a diffraction grating with a period greater than the wavelength with subwavelengthstructures. Those can, in a first step, be considered as an effective medium with different effective indices for TE- and TM-polarization, which is the so-called form birefringence $[5,6]$. The final structure can be seen in Fig. 1. It realizes a phase shift of $\phi_{T M}=\pi$ and $\phi_{T E}=2 \pi$. The initial design has been laid out with the Effective Medium Approach with correction terms for larger structures [5] and then refined with RCWA-simulations. With the parameters height, fill factor and material, the desired phase difference $\Delta \psi$ is tunable.

\footnotetext{
*e-mail: julian.wuester@tu-ilmenau.de
}

Simulations show that the high phase difference $\Delta \psi=\pi$ within the effective medium mainly relies on the refractive index $n_{g}$. Due to manufacturing restraints in terms of the aspect ratio (see Section 3) we choose the highly refractive material silicon and a design wavelength of $\lambda=1400 \mathrm{~nm}$. The structure sizes are $p_{1}=500 \mathrm{~nm}, p_{2}=10 \mu \mathrm{m}$, the fill factor $f=0.5$ and the etching depth $h=700 \mathrm{~nm}$.

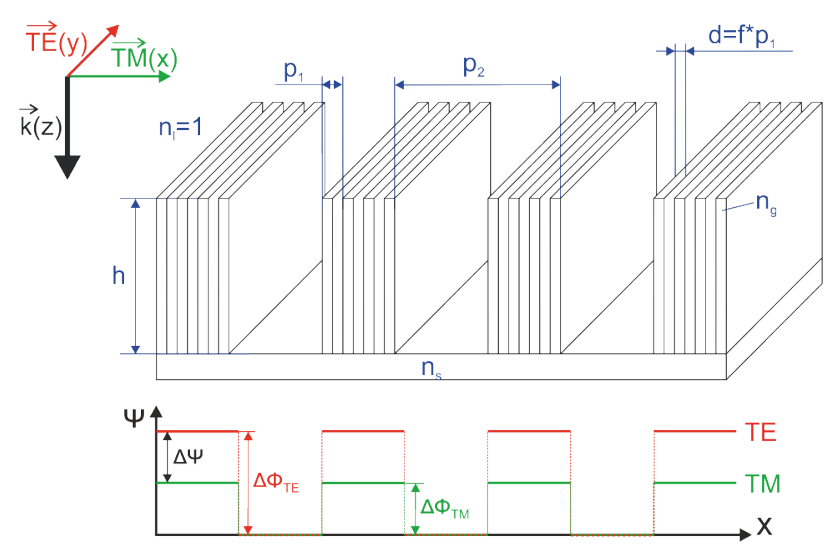

Figure 1. Design layout

\section{Manufacturing}

Due to the potential cost-efficiency (reproducability of large structured areas) in comparison to direct-writing techniques, Soft-UV-Nanoimprint-Lithography was chosen for the manufacturing process. The main limitation within the design is the limited aspect ratio one can expect from the lithographic process, which also limits our choice of the fill factor. Therefore various steps are needed to manufacture the grating in the high-refractive silicon. 
We started from an electron-beam-structured chromium mask on fused silica, which was fabricated externally. All the other steps were carried out in-house.

The first dry-etching pattern transfer of the chromium layer to the fused silica wafer $\left(\mathrm{RIE} \mathrm{CHF}_{3} / \mathrm{SF}_{6}\right)$ results in our master for the following NIL-steps. The master with structure heights of about $250 \mathrm{~nm}$ is coated with FDTS for anti-sticking. The diluted PDMS is poured onto the master and after hardening we yield the PDMS stamp. We chose a $\mathrm{SiO}_{2}$ hard mask. For that we executed a dry thermal oxidation process with a silicon wafer up to an oxide layer thickness of $200 \mathrm{~nm}$. On top of that we spincoat the NIL-resist NIL 210. First we execute the imprinting process with the again anti-sticking-treated stamp to reproduce the structures in the resist layer. The master can be reused. Two more etching steps are needed to come to the final structure in silicon, which can be seen in Fig 2. The transfer from the resist pattern to the oxide layer is carried out with a RIE process $\left(\mathrm{CHF}_{3} / \mathrm{AR}\right.$ PlasmaPro 100) and the transfer from the oxide mask to the silicon wafer with ICP-RIE $\left(\mathrm{SF}_{6} / \mathrm{O}_{2}\right.$ PlasmaPro 100 Cobra). The most critical step due to the different scales $(\mathrm{nm} / \mu \mathrm{m} / \mathrm{cm})$ within the whole process is the molding of the PDMS stamp.

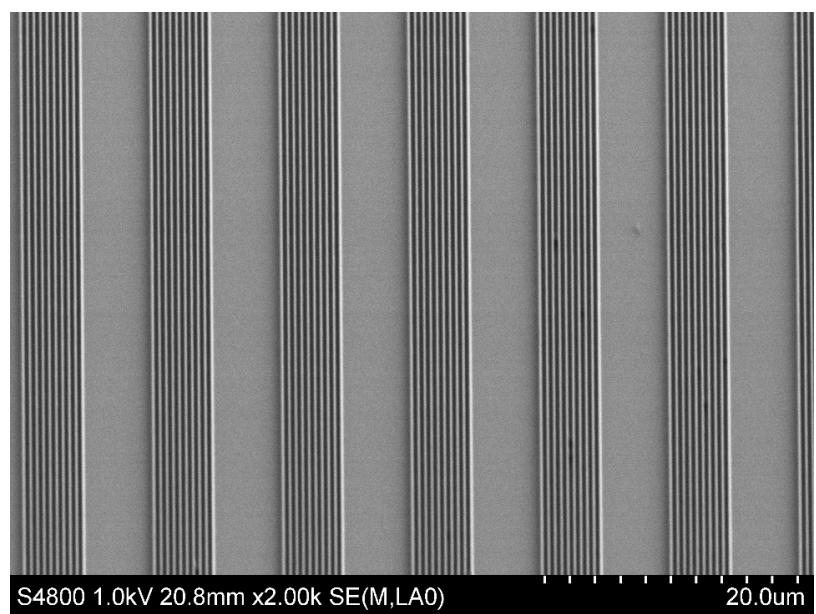

Figure 2. SEM image of the grating in silicon

\section{Experimental results}

We used an SC fiber laser, set to a wavelength of $\lambda=1400 \mathrm{~nm}$. The fiber is not polarization-maintaining, so we let the light pass through a linear polarizer before interacting with the grating from the back side of the polished waver. The intensities are measured with Thorlabs photodiodes S122C. For obtaining the different polarization states just the polarizer is rotated. The results can be seen in Fig. 3. Note that due to the refractive index $n=3.49$ the Fresnel losses are very high. About $50 \%$ of the light is lost in transmittance. The results show the diffraction efficiencies relative to the intensity of the incident light under an angle of $0^{\circ}$, including the Fresnel losses. This means that the efficiencies could be significantly increased with a lower refractive material or anti-reflection coating. Also experiments were carried out with varying wavelengths and varying incidence angles. Within a range of $1400 \mathrm{~nm} \pm 60 \mathrm{~nm}$ there is no significant change in efficiency.

Measuring the intensities in relation to the incidence angle shows, that the grating is operable within a range of $0^{\circ} \pm 30^{\circ}$, as the efficiency contrast between TE and TM is still very high.

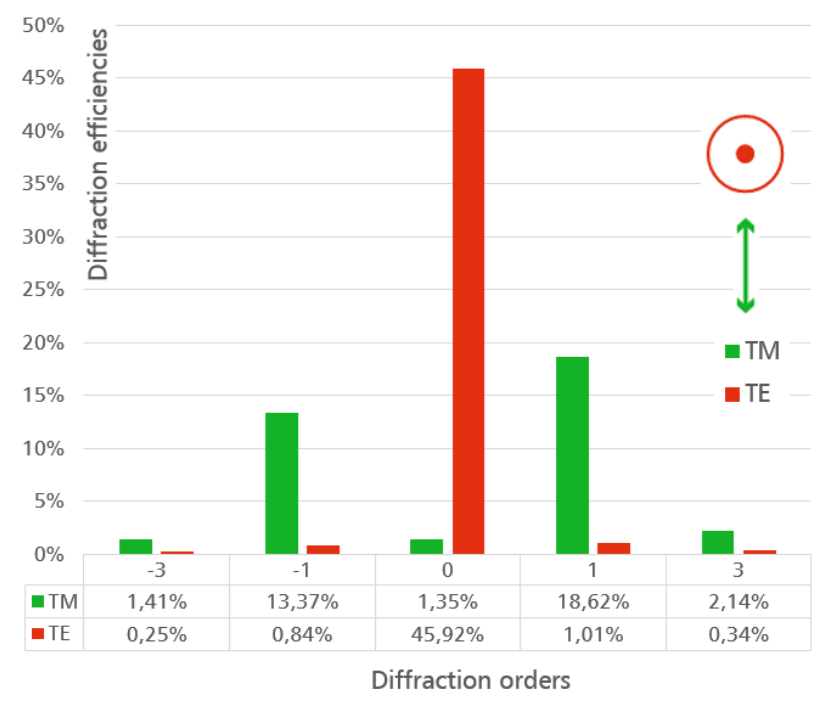

Figure 3. Diffraction efficiencies

\section{Summary and Outlook}

We presented an approach for the design of diffractive optics with polarization-dependent diffraction efficiencies. As an example we chose a binary grating with subwavelenghth-structures which was fabricated with Soft-UV-Nanoimprint-Lithography. It acts as a polarizing beamsplitter over a broad range of incidence angles and with tolerance in wavelength or etching depth, respectively. The design approach to combine those subwavelength gratings with diffractive optics with conventional dimensions is also applicable for multilevel elements and is therefore a promising way to add new functionalities to DOEs.

\section{References}

[1] H. Bartelt et al., Optik 112, 283-288 (2001)

[2] P. Lalanne et al., J. of Optics A 215, 214-219 (1999)

[3] M. Bichra et al., Appl. Optics 56, A13-A17 (2017)

[4] M. Bichra et al., Appl. Optics 57, 3808-3816 (2018)

[5] H. Haidner, Dissertation (FAU, Erlangen, 1994)

[6] P. Lalanne et al., J. Modern Opt. 43, 2063-2085 (1996)

[7] J. Wüster et al., EOS Diffractive Optics, Jena (2019) 